\title{
A Literature review of Routing and Cloud Deployment Methodologies used in an Air Vehicle
}

\author{
Vivekanadam Balasubramaniam, \\ Faculty of Computer Science and Multimedia, \\ Lincoln University College, \\ Kota Bharu, Malaysia. \\ Email: vivekanandam@lincoln.edu.my
}

\begin{abstract}
We present a complete overview of routing protocols, routing algorithms, path planning, and cloud deployment for vehicle navigation in several fields of study in this article. In this article, we compare several approaches and algorithms with the goal of identifying the best feasible ones based on the type of application being utilized. In general, navigation of vehicles will be based on models and methods. Hence in this paper each characteristics are examined in detail and the research has been done accordingly. Under each characteristic, performance evaluation criteria are separately analysed. Questions are also provided for which the literature review serves as a form of discussion, according to the research challenge and criteria. For path planning, node-based as well as traditional algorithms are considered as the best choices. Similarly, the performance is significantly improved when using hybrid routing protocols and route planning methodologies that prefer graph based techniques. It has been observed that, a number of future research directions such as routing algorithm with queuing theory and path planning with critical link methods also serve the probable domains. This work is a concise comprehensive study of the various characteristics of a vehicle with respect to navigation. A comparison of techniques, algorithms and methods by using the standard performance criteria has also been elaborated.
\end{abstract}

Keywords: Challenge-response, electronic control units, integrity, vehicular network, blockchain 


\section{Introduction}

Air vehicles are commonly developed for the purpose of military applications. Over the last few decades, many air vehicles have been used for the purpose of urban surveillance by means of precision agriculture and in many civil applications. A number of these applications require many such air vehicles as they involve high scalability, simultaneous operations and multitasking. The drawback is that using multiple air vehicles is a highly demanding research area, which is complex with additional computational cost, when compared to a single air vehicle [1]. Control, navigation and guidance are the major research areas in a vehicle operation. Number of research workers currently carried out the means of vehicle operation and the research activities can be categorised into routing protocols routing algorithms and path planning [2]. This work is an attempt to represent a complete research by providing an overview of the many components of our navigation, as well as the routing protocols and route planning methodologies employed. A detailed study of the performance of the evaluated works is also provided in order to establish the best functioning model. In [3], the authors have reviewed the 3D (D represents degree of freedom) oath planning methodology. Similarly, the authors in [4] have presented a comparative note on the different routing protocols that exist along with their drawbacks. A comparative survey of various path planning algorithms are described in [5], while [6] shows the various ad-hoc net routing strategies involved in classifying the air vehicles.

The already existing survey work has not provided a complete record for comparing the various techniques and algorithms based on the performance criteria [7]. To overcome this drawback, the main aim is to realise a comprehensive study on the various navigation aspects of the air vehicles and further compare it with equivalent methodologies to determine the optimal mode of operation based on the results recorded [8]. The ultimate aim of this work is to provide exclusive guidelines for the researchers with the help of performance comparison so that based on the application and requirement; they will be able to choose the appropriate characteristics for their research work. Potential future research work is also briefly discussed according to the performed analysis. The rest of the work can be organized as follows [9]. The next session gives a brief overview of the methodology selected in this paper. The various path planning algorithms are detailed in the next session followed by using the 
routing algorithms and routing protocols. A comparison is drawn on the algorithms and methods used with definitive answers described in section 6. Finally a conclusion is drawn in section 7 according to the observed results [10].

\section{Methodology}

A complete review of the various works in the field of vehicle navigation is made in this work to select the best methodology. The crucial steps in methodology are formulation of research questions and performance evaluation [11].

\subsection{Performance evaluation criteria}

This paper aims to provide a comparative study of techniques, algorithms and methods that are used by air vehicles for the purpose of navigation [12]. For routing protocols, routing algorithms and path planning, many methods are compared and analyzed. Table 1 gives a summary of the performance criteria for the different categories studied.

\subsection{Research questions}

The primary objective of the proposed methodology is to comprehend the various crucial aspects of air vehicle navigation using an elaborate comparison of the various methods and algorithms used. Also, this paper has analyzed the different methodologies [13] in order to determine the best algorithm for specific applications. The following research questions provide a brief view of the work's aim.

1. RQ1. What are the probable areas in research of multiple or single air vehicle working?

2. RQ2. Which routing protocol, routing algorithm and planning algorithm is the best choice?

3. RQ3. What is the performance of each routing protocol, routing algorithm and planning algorithm based on performance criteria? 
Journal of Artificial Intelligence and Capsule Networks (2021)

Vol.03/ No.02

Pages: 113-124

http://irojournals.com/aicn/

DOI: https://doi.org/10.36548/jaicn.2021.2.004

\section{Air Vehicle Three Degree-of-Freedom Path Planning}

To choose the optimal path from source to destination, path planning plays a crucial role by considering all the requirements and constraints involved in vehicle navigation. Over the past few decades, the field of path panning has grown substantially with a large portion in 2D planning. However, the use of 2D planning will not be appropriate to provide effective solutions, when used in 3D domains [14]. On the other hand, 3D planning will enable a positive growth by considering a number of constraints like temporal, physical and geometric constraints and ambiguities. A summary of the 3D path planning is also discussed in this work. Fig. 1 shows the different categories which fall under 3D path planning algorithms [15].

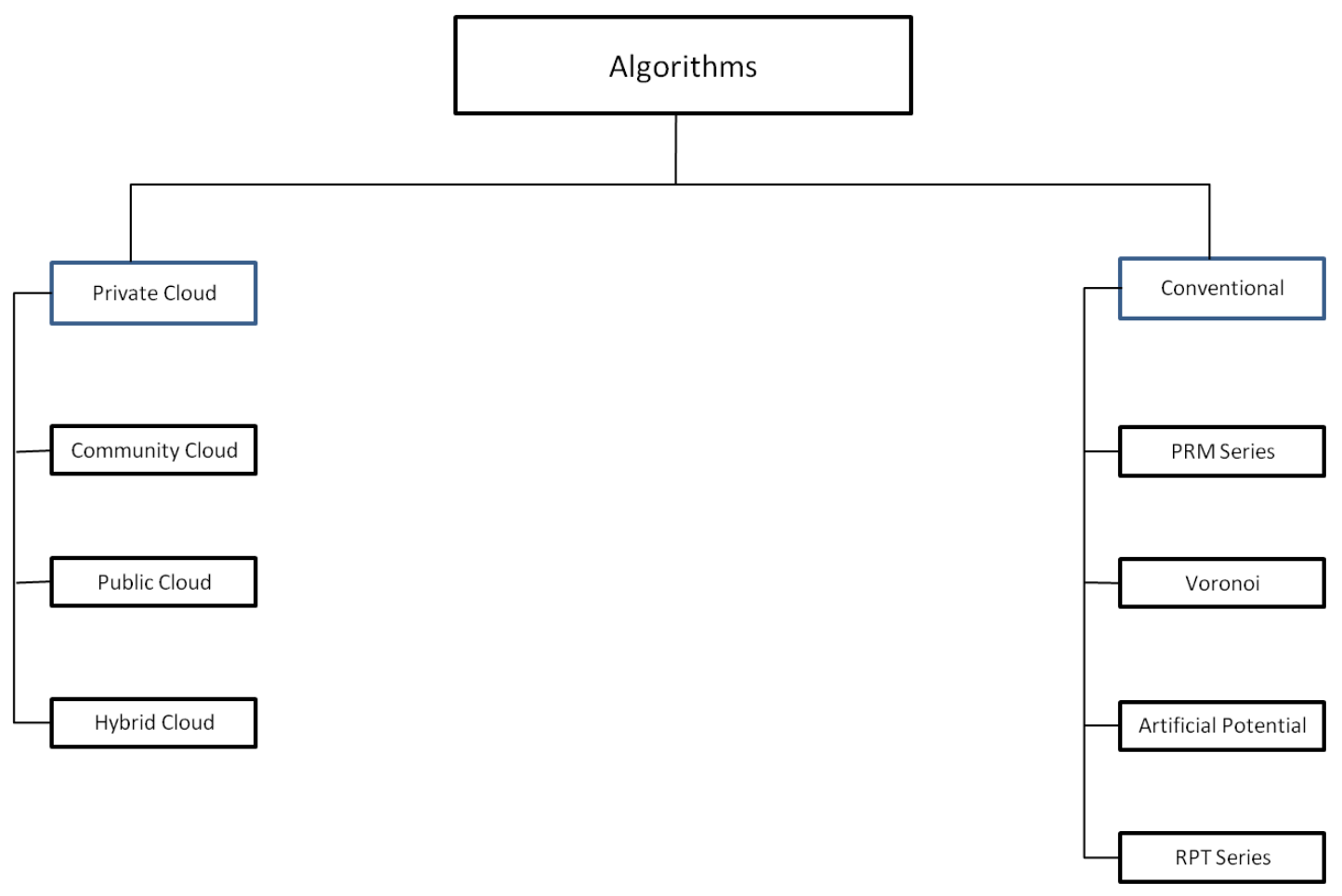

Fig.1. Algorithms used in Path Planning and Cloud Deploying 


\section{Conventional Algorithms}

Information of the workspace needs to be represented mathematically using the conventional algorithms. The conventional algorithm can also be classified into passive and active algorithms.

\subsection{PRM Series Algorithms}

In 1994, PRM series of algorithm was incorporated in the 3D environment by Kavraki and Latombe [16] resulting in delivering a fast exploration performance. This was also elaborated by using an obstacle-based nodes [17] generation strategy by Amato et al. in [18]. At each step, the road map candidate points are selected on the obstacle surface.

\subsection{Voronoi Algorithms}

The Voronoi diagram was introduced by the Shamos and Hoey (1975) to introduce the computational geometry field. Similarly, a 3D Voronoi diagram was proposed by Lucknikov et al and can be used to determine the process of path planning in 3D complex system. Using Dijkstra's algorithm, geographical information system, Liu and Zhang used this algorithm to identify the shortest path [19].

\subsection{Artificial Potential Algorithms}

Due to the low computational complexity, potential field methods are commonly researched and incorporated globally. When using artificial potential algorithm, it is possible to allocate free space to allow the air vehicle to be initiated similar to the reaction of particles in a potential field cause of force [20].

\subsection{Rapidly-Exploring Random Trees (RRT) Series Algorithms}

The following are the algorithms based on RRT series and they are discussed below.

- The tree version of Rapidly-Exploring Random Graph (RRG) is the Rapidlyexploring Random Trees-Star (RRT), which is used to handle differential constraints, keeping the RRG's asymptotic optimal property intact; the bad connections are 
removed by using the RRT, which helps to enhance the solution and substantially reduce its cost.

- Though the rapid exploring random graph, RRT promises to deliver completeness and has an overall positive performance, where it doesn't consider the result quality. It is also worth noting that, the RRT algorithm is not asymptotically optimal. Hence, the RRG methodology is introduced to enable optimality in an asymptotic environment.

- Voronoi bias problem is common in RRT and was solved by the Dynamic Domain Rapidly-Exploring Random Tree (DDRRT) algorithm. This algorithm enables quick exploration. The drawback is that this method is similar to that of a typical RRT when post-smooth processing is involved. The path created by the DDRRT algorithm is not a beneficial path.

- Lavalle in 1998 introduced the rapidly-exploring random trees RRT. This methodology was introduced to handle path planning issues like kino-dynamic, nonholonomic and holonomic constraints. The advantage of this methodology is that it can handle many DOF problems simultaneously.

\section{Deployment Models}

- Hybrid Cloud: More than one distinct cloud infrastructures (public, community or private) are put together through proprietary or standardized technology to enhance application and data portability will form the hybrid cloud.

- Public Cloud: One type of cloud infrastructures that gives access to the public is known as public cloud. It could be operated, managed or owned by a government, academic or business organisation in a combined form. This type of architecture will be available in the cloud provider environment.

- Community Cloud: The community cloud is dedicated to provide exclusive service to customers from a specific organization with a specific concern. It could be operated, managed or owned by one or more than one organization such as third party, community or even a combination of it. It could also be located on either the premises or away from it.

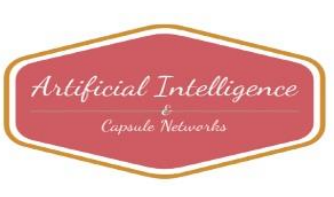


- Private Cloud: This infrastructure is designed to provide an exclusive environment from a particular organization, which has been held by many consumers. It could be operated, managed or owned by a third party, organization or a combination of the two.

\section{Performance Evaluation}

\subsection{Routing Protocol}

Fig.2 shows the different types of routing protocols that are used to determine the path in which the air vehicle is to travel.

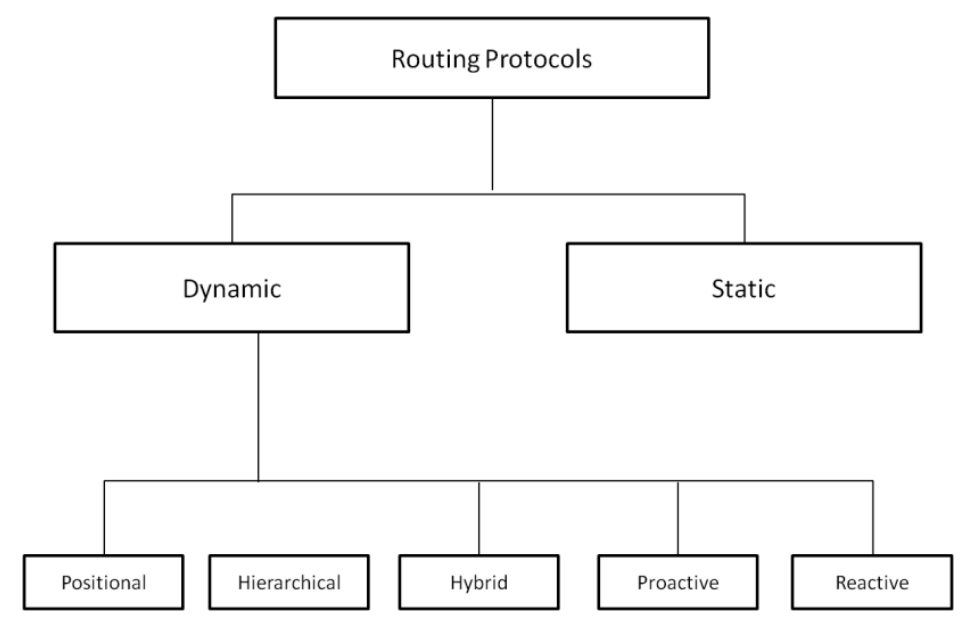

Fig.2. Types of Routing Protocol

Table 1 showcases the performance evaluation of the routing protocols based on the type of environment in which it is incorporated. It indicates that, these protocols do not have tolerance for fault, are not load optimized and do not have enough energy. Position-based protocols are in need of the system location in order to determine the optimal shortest path towards destination. The dynamic protocol is used in reactive protocols, which require frequency updates in an ever-changing environment. At a regular basis, the proactive protocols are used and are not used in the dynamic environment. Moreover, their inefficient

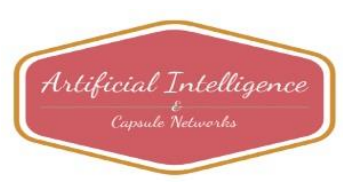


Journal of Artificial Intelligence and Capsule Networks (2021)

Vol.03/ No.02

Pages: 113-124

http://irojournals.com/aicn/

DOI: https://doi.org/10.36548/jaicn.2021.2.004

energy usage also leads to an increase in the overall cost analysis of the system. The advantages of these protocols are combined to form the hybrid protocol. When sparse and large networks are concerned, the hierarchical protocols are considered as the best option, since it can be used with many optimization parameters.

Table 1. Routing Algorithm Performance Evaluation

\begin{tabular}{|c|c|c|c|c|c|c|}
\hline $\begin{array}{c}\text { Routing } \\
\text { algorithm }\end{array}$ & CT & RC & Adaptiveness & IC & OE & Complexity \\
\hline $\begin{array}{c}\text { Queuing } \\
\text { Theory }\end{array}$ & High & Separate & Yes & High & Static & Low \\
\hline Deterministic & Mild & Separtate & No & High & Dynamic & Medium \\
\hline Probabilistic & Mild & Mix & Mix & High & Static & Medium/High \\
\hline Graph & Moderate & Mix & No & Low & Static/Dynamic & Low \\
\hline
\end{tabular}

\subsection{Performance Evaluation of 3D Path Planning \& Routing Protocol}

Various 3D path planning algorithms and their performance evaluation are listed in the table below based on the Fault Tolerance (FT), Operation, Adaptiveness and Complexity parameters.

Table 2. Performance of 3D Planning Algorithm

\begin{tabular}{|c|c|c|c|c|}
\hline 3D path planning algorithm & FT & Operation & Adaptiveness & Complexity \\
\hline \multicolumn{5}{|l|}{ Learning Based } \\
\hline $\mathrm{NN}$ & No & Static/Dynamic & No & Low \\
\hline Evolutionary & Yes & Static & Yes & Medium \\
\hline Hybrid & Yes & Static & Yes & Low \\
\hline \multicolumn{5}{|l|}{ Model based } \\
\hline Linear & No & Static/Dynamic & No & Medium \\
\hline \multirow[t]{2}{*}{ Optimal control } & Yes & Dynamic & Yes & High \\
\hline & & Static & & \\
\hline \multicolumn{5}{|l|}{ Cell Based } \\
\hline A star & Yes & Dynamic & Yes & Medium \\
\hline D star & Yes & Dynamic & Yes & Low \\
\hline Dijksta & No & Static/Dynamic & No & Low \\
\hline \multicolumn{5}{|l|}{ Conventional } \\
\hline RRT & No & Static/Dynamic & No & Low \\
\hline Artigicial Potential & Yes & Static /Dynamic & Yes & Medium \\
\hline PRM & Yes & Dynamic & Yes & Low \\
\hline
\end{tabular}


It is observed in Table 2 that, the cell-based and conventional algorithm showcases the similar outputs. Hence they are considered as the best options, when it comes to easy implementation and quick convergence. However, the drawbacks with these two algorithms are their fault tolerance and lack in adaptivity, which will result in distortion with other requirements that need to be addressed. This indicates that, the model-based algorithm is the only appropriate alogorithm when dynamic constraint occurs and will also require the investment of cost function. However, this model cannot be executed in real time and it is also complex in nature. Many advantages are observed in the learning based algorithms, they are fault tolerance, adaptivity and low complexity. However, the biggest drawback is the requirement of financial aspects in order to incorporate the complex methodology, which is not appropriate for small appliances. A combination of learning-based algorithm and cellbased methodology results in developing a hybrid algorithm.

\section{Conclusion}

One of the emerging fields that has gained increased research attention in the past few years are air vehicle operations and planning. In this paper, a detailed review has been made on the routing protocols, routing algorithms, path planning algorithms and cloud architecture. This is followed by a comparative study on various protocols and algorithms, which are evaluated to determine the performance and credibility of each system. Accordingly, it has been identified that, when the device to be built requires less number of components, cell-based path planning is used. Similarly, hybrid algorithm and learning-based algorithms are considered to be more appropriate for large and complex networks. In recent years, vehicles are using hybrid protocols to provide more positive aspects when compared with the other similar protocols. Some of the possible future scope that can be implemented in the near future are the incorporation of satellite communication for the betterment of path planning and the use of improved routing algorithms with a cost-effective methodology for larger networks. 
Journal of Artificial Intelligence and Capsule Networks (2021)

Vol.03/ No.02

Pages: 113-124

http://irojournals.com/aicn/

DOI: https://doi.org/10.36548/jaicn.2021.2.004

\section{References}

[1] Adithya, M., P. G. Scholar, and B. Shanthini. "Security Analysis and Preserving Block-Level Data DE-duplication in Cloud Storage Services." Journal of trends in Computer Science and Smart technology (TCSST) 2, no. 02 (2020): 120-126.

[2] Chamam, A., \& Pierre, S. (2009). On the planning of wireless sensor networks: Energy-efficient clustering under the joint routing and coverage constraint. IEEE Transactions on Mobile Computing, 8(8), 1077-1086.

[3] Suma, V., and Wang Haoxiang. "Optimal Key Handover Management for Enhancing Security in Mobile Network." Journal of trends in Computer Science and Smart technology (TCSST) 2, no. 04 (2020): 181-187.

[4] Nohl, A. R., \& Molnár, G. (2003). The convergence of the OSPF routing protocol. Periodica Polytechnica Electrical Engineering (Archives), 47(1-2), 89-100.

[5] Adithya, M., P. G. Scholar, and B. Shanthini. "Security Analysis and Preserving Block-Level Data DE-duplication in Cloud Storage Services." Journal of trends in Computer Science and Smart technology (TCSST) 2, no. 02 (2020): 120-126.

[6] Rahman, T. R., \& Patil, N. B. (2018). EVALUATION OF MOBILE NETWORK PLANNING STRATEGY WITH ROUTING PROTOCOL SUPPORT. International Journal of Advanced Research in Computer Science, 9(1).

[7] Suma, V. "Wearable IoT based Distributed Framework for Ubiquitous Computing." Journal of Ubiquitous Computing and Communication Technologies (UCCT) 3, no. 01 (2021): 23-32.

[8] Hosahalli, D., \& Srinivas, K. G. (2019). Cross-layer routing protocol for event-driven M2M communication in IoT-assisted Smart City Planning and Management: CWSNeSCPM. IET Wireless Sensor Systems, 10(1), 1-12.

[9] Adam, Edriss Eisa Babikir. "Survey on Medical Imaging of Electrical Impedance Tomography (EIT) by Variable Current Pattern Methods." Journal of ISMAC 3, no. 02 (2021): 82-95.

[10] Baziyad, M., Saad, M., Fareh, R., Rabie, T., \& Kamel, I. (2021). Addressing Real-Time Demands for Robotic Path Planning Systems: A Routing Protocol Approach. IEEE Access, 9, 38132-38143.

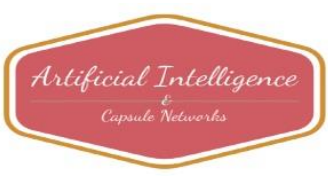


Journal of Artificial Intelligence and Capsule Networks (2021)

Vol.03/ No.02

Pages: 113-124

http://irojournals.com/aicn/

DOI: https://doi.org/10.36548/jaicn.2021.2.004

[11] Adam, Edriss Eisa Babikir. "Evaluation of Fingerprint Liveness Detection by Machine Learning Approach-A Systematic View." Journal of ISMAC 3, no. 01 (2021): 16-30.

[12] Wang, Z., Feng, X., Qin, H., Guo, H., \& Han, G. (2017). An AUV-aided routing protocol based on dynamic gateway nodes for underwater wireless sensor networks, 18(2), 333-343.

[13] Manoharan, J. Samuel. "Capsule Network Algorithm for Performance Optimization of Text Classification." Journal of Soft Computing Paradigm (JSCP) 3, no. 01 (2021): 1-9.

[14] Liu, L. and Zhang, S. (2009), "Voronoi diagram and GISbased 3D path planning", 17th International Conference on Geoinformatics, Geoinformatics 2009, available at: https://doi. org/10.1109/GEOINFORMATICS.2009.5293350.

[15] Manoharan, Samuel, and Narain Ponraj. "Analysis of Complex Non-Linear Environment Exploration in Speech Recognition by Hybrid Learning Technique." Journal of Innovative Image Processing (JIIP) 2, no. 04 (2020): 202-209.

[16] Kavraki, L.E. and Latombe, J.-C. (1994), "Randomized preprocessing of configuration for fast path planning”, IEEE International Conference on Robotics and Automation (ICRA), pp. 2138-2145.

[17] Adam, Edriss Eisa Babikir. "Deep Learning based NLP Techniques In Text to Speech Synthesis for Communication Recognition." Journal of Soft Computing Paradigm (JSCP) 2, no. 04 (2020): 209-215.

[18] Amato, N.M., Bayazit, O.B. and Dale, L.K. (1998), “OBPRM: an obstaclebased PRM for 3D workspaces", 3rd Workshop on the Algorithmic Foundations of Robotics on Robotics: The Algorithmic Perspective: The Algorithmic Perspective (WAFR '98), pp. 155-168.

[19] Bashar, Abul. "Sensor Cloud Based Architecture with Efficient Data Computation and Security Implantation for Internet of Things Application." Journal of ISMAC 2, no. 02 (2020): 96-105

[20] Lavalle, S.M. (1998), "Rapidly-exploring random trees: a new tool for path planning", Techreport, Vol. 11, available at: https://doi.org/10.1.1.35.1853. 
Journal of Artificial Intelligence and Capsule Networks (2021)

Vol.03/ No.02

Pages: 113-124

http://irojournals.com/aicn/

DOI: https://doi.org/10.36548/jaicn.2021.2.004

\section{Author Biography}

Vivekanadam Balasubramaniam is currently working as professor in the Faculty of Computer

Science and Multimedia in Lincoln University College at Kota Bharu, Malaysia. His area of research includes machine learning, deep learning, neural network architectures, cloud computing, blockchain technologies, network engineering, robotics, embedded engineering, biomedical instrumentation and social networks. 